\title{
Data security and Influence of Cloud Computing in Electronic Commerce Industry
}

\author{
Sanghita Roy \\ Institute of Business Management \& Research \\ 65/25 Jyotish Roy Road, New Alipore \\ West Bengal, India \\ Pin-700053
}

\author{
Indrajit Sinha, Ph.D \\ Bharatiya Bidya Bhavan, \\ Institute of management Science \\ 91, Salt Lake City, Karunamoyee \\ West Bengal, India \\ Pin - 700109
}

\begin{abstract}
Cloud computing is an emerging computing technology that uses Internet and central remote servers to maintain data and applications. Cloud computing allows customers and businesses to use applications without installation and access their personal files or data at any corner of the world with Internet access. Presently different types of browser based applications are running in cloud computing environment. Electronic commerce is major of them. This paper has discussed the concepts of cloud computing and on the basis of this issue analyzes its impact on E-commerce industry. Secondly, from the characteristics of cloud, network security problems and solutions are also discussed. Cloud computing has to effectively manage security and privacy issues of cloud applications. Based on literature this paper has discussed how traditional E-commerce businesses and industries are influenced by cloud computing and provide an overall security perspective of cloud computing with the aim to highlight the security concerns.
\end{abstract}

\section{Keywords}

Cloud computing, E-commerce, Network security, Public cloud, Private cloud, Hybrid Cloud, policies, cloud security

\section{INTRODUCTION}

Since it was proposed by Google in 2007 [1], at present cloud computing is not a new concept to most tech-savy people. Cloud computing is a new and emerging information technology innovation that brings the concept of virtualization of data storage, local networks (infrastructure) as well as software. [2,3] The "cloud" is a metaphor - it is an abstraction hiding the complex infrastructure of the Internet. It is a low-cost viable option to the users in which IT-related capabilities are provided "as a service", allowing users to access technology-enabled services from the Internet without knowledge of or control over the technologies behind these services. The survey (done by International Data Corporation (IDC) in 2008-2009) results showed that cloud computing is best suited for those developers or research projects and even E-commerce entrepreneurs who are seeking a quick solution for startup and want to keep IT budget to a bare minimum. [4] Some experts evaluated the effects of cloud computing is superior than Electronic commerce. E-commerce which includes retail shopping, banking, auctions, air ticket booking nearly anything you can imagine in the real world brings huge profit to the enterprises. However, E-commerce costs are huge. Cloud computing gives new opportunities to Ecommerce companies where they do not have to spend a lot of manpower, financial and material resources to set up ebusiness system and maintenance of background software. All the tasks can be handled by cloud computing providers.
On the other side, companies can give attention to improve customers' loyalty and enterprise benefit. [5]

Cloud computing can be broken down into three segments"applications", "platforms" and "infrastructures". Each segment serves a different purpose and offers different products for businesses and individuals around the world. Cloud computing can be seen as a Service-OrientedArchitecture (SOA) which utilizes software as a service (SaaS), such as Web 2.0 and other technologies. For example - Google apps provides common online business applications that are accessed from a web browser, while the software and data are stored on Internet server.

In order to minimize potential security trust issues as well as adhering to government issues facing cloud computing, a prerequisite control measure is to ensure that a concrete cloud computing Service Level Agreement (SLA) is put in place and maintained when dealing with outsourced cloud service providers and specialized cloud vendors. Currently cloud computing clients have to trust on third party cloud providers on many aspects, especially on the availability of cloud services as well as data security. [6]

As a new IT concept, cloud computing is being applied to many industries specially Electronic commerce industry. This paper discusses the impacts of cloud computing on traditional E-commerce from the perspective of technology, service and industry chain and provides suggestions on improvement of e-commerce business in the cloud era. [1] At the same time this paper highlights security measures of cloud computing.

\section{CLOUD COMPUTING LITERATURE REVIEW}

Before going into the depth of literature primarily we have to know what is Cloud Computing? Since last decade there is a steady shift from traditional software models to Internet software applications which are always bit complicated and expensive. A group of expert people are required to develop, install, configure, test, run and update the system. With cloud computing, those headaches can be easily eliminated because that's the responsibility of an separate experienced vendor like salesforce.com who will execute the service. Clients only have to pay for their need, upgrades are automatic and uploading or downloading is easy. [19] There are many definitions of cloud computing based on various criterion. Gartner defines cloud computing as a style of computing in which scalable and elastic IT-enabled capabilities are delivered as a service using Internet technologies. Wikipedia defines - Cloud computing is a phrase used to describe a variety of computing concepts that involve a large number of computers connected through a 
real-time communication networksuch as the Internet. In brief cloud computing can be defined as a computing paradigm where services and data reside in shared resources in scalable data centers and those services and data are accessible by any users through authenticated device over Internet. [7]

Almost free of cost cloud-based apps can be used anytime and anywhere. For that users only have to open a browser, $\log$ in, customize the apps and start using it. [19] Businesses are running different kinds of apps in cloud like Customer Relationship Management, HR, accounting and much more after rigorous testing of security and reliability of the infrastructure. The latest highly popular consumer apps are Facebook, Whatsapp and Twitter.Cloud computing customers don't generally own the physical infrastructure on which apps run and store data. Rather, they rent usage from a third party provider and they use the system as they need it.

The objective of our paper is to judge the influence of cloud computing on E-commerce. Since its emergence, influences of cloud computing has been discussed in different literature by various authors. In rural China lack of teaching resources hindered their educational growth. Li J. and Liu J. [8] analyzes the influence of cloud computing in Chinese educational information system. Shuai Q. [9] describes the troubles faced by Chinese m-learning, which includes limited resources of mobile devices, limited storage capacity and expensive investment of hardware and software. Cloud computing, due to its reliability and easy customization facility can solve the problem. However all these possible benefits are theoretically discussed rather than examined empirically. Kashefi, F., et al [10] emphasizes the technical impact of cloud computing on big companies and organizations, such as Google in their adoption of new methodology, techniques and analyses. Lai, S. [11] analyses the impact of cloud computing on traditional software project and also offers application of migration strategies, security strategies and corresponding migration tools for software project development. Along with rapid development of economic environment, virtual operation performance management is becoming more important. Zhang, H. [12] figures out that cloud computing technology will become the best choice of the virtual operation process due to its features such as reliability and security and it can be applied in different levels and different aspects of performance management.

There are two drawbacks of this literature study. Firstly, the present literature focuses on one or two aspects of cloud computing impacts of a special area. Secondly, there are few literature mentioning the influences of cloud computing on electronic commerce and also security issues. Actually, the rapid development of E-commerce requires involvement of cloud computing into its technical architecture, business mode and service mode. In the first part of the paper security issues of cloud computing is discussed and then effect of cloud computing on electronic commerce industry is explained

\section{FEATURES OF CLOUD COMPUTING}

(a) Centralized infrastructure in certain location with lower costs.

(b) Improve efficiency of unutilized system for maximum utilization (c) Reliability is improved as multiple redundant sites are used which makes well designed cloud computing

(d) Apps can be easily purchased and billed by consumption

(e) Easy access over the Internet by any device

(f) Easy maintenance because they do not need to be installed on each users' computer

(g) Security could improve due to centralization of data. Though security is often as good as or better than traditional system, but there is chance of loss of control over certain sensitive data. However complexity of security increases when data is vastly distributed and greater number of devices and multi-tenant systems are being shared by unrelated users. Private cloud installation can retain control over the infrastructure and avoid losing control of information security.

\section{CLOUD COMPUTING DEPLOYMENT MODEL}

In order to provide a secure cloud computing solution, it is vital to decide which type of cloud to select. There are three types of cloud deployment models.

Public cloud - Public cloud is ideal for businesses seeking less complex IT hosting and no complicated contracts. It allows users' access to the cloud via interfaces using mainstream web browsers. It is typically based on pay-peruse model, similar to a prepaid electric meter. Applications run on public cloud have either seasoned demand or unpredictable traffic. It is less secure than other cloud models.

Private cloud - Private cloud is designed with organization's internal enterprise data center. Here scalable resources and virtual applications given by the cloud vendors are pooled together and available for cloud users to share and use. Just like Intranet, all the resources and applications are managed by organization itself. Only the organization people and designated stakeholders may have access to operate on a specific private cloud. [13] Thus, private cloud is much more secure than public cloud.

Hybrid cloud - Hybrid cloud is a mix of both public and private cloud which is centrally managed and circumscribed by a secure network. [14] It provides more secure control of the data and applications and allows various parties to access information over the Internet.

Therefore, in order to decide which type of cloud to deploy, business managers' need to access the security considerations from an enterprise architectural point of view.

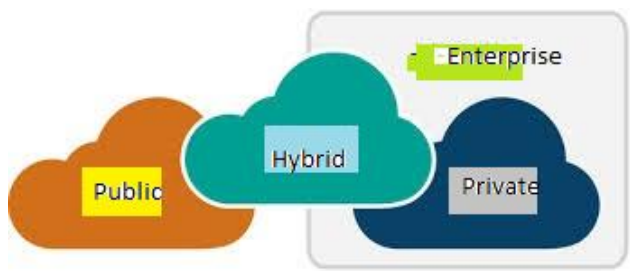

Figure 1: Cloud Computing Deployment Model 


\section{CLOUD COMPUTING DELIVERY MODEL}

After cloud deployment models, based on security there are three types of cloud delivery models. The degree of information security is positively correlated with industry standards and legislations among cloud stakeholders. Delivery models are as follows-

(a) Infrastructure as a Service (IaaS) Infrastructure as a Service is a single layer cloud where cloud computing vendor's dedicated resources are only shared with contracted clients at pay-per-use basis. As a result, initial investment cost of computer servers, networking devices, processing power etc. are minimized. IaaS also allows varying degrees of financial and functional flexibility which is not found in internal data centers or with co-location services, because computing resources can be added or released much more quickly and cost-effectively than in an internal data center. [15]

(b) Software as a Service (SaaS) - Software as a Service is based on pay-per-use costing model where software applications are leased out to contracted organizations by specialized SaaS vendors. Initially software has limited functionality, it can be easily customized based on demand which is billed accordingly. SaaS providers may host the software either in their own data center or outsourced to IaaS providers. [16] Softwares are accessed using secured web browser over the Internet. Web services (WS) security, XML encryption, Secure Socket Layer (SSL) etc is used in enforcing data protection transmitted over the Internet.

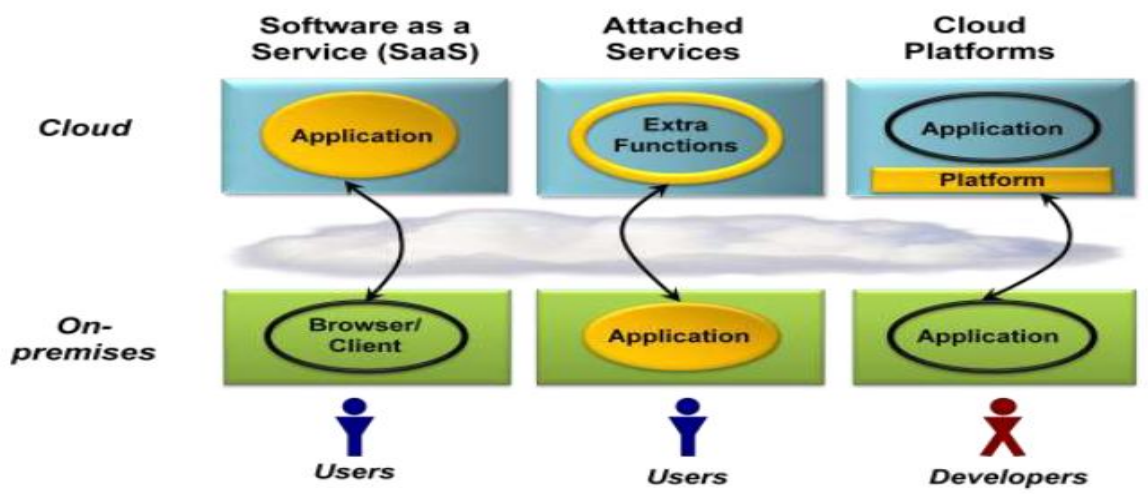

Figure2: Cloud Computing Delivery Model

(c) Platform as a Service (PaaS) - Function of Platform as a Service cloud layer is similar to IaaS with an addition "rented" functionality. PaaS services are costlier than IaaS and SaaS. Use of virtual machines act as a catalyst in the PaaS layer in cloud computing. Virtual machines are protected against malicious attacks such as cloud malware.

In a virtual environment physical resources, infrastructures as well as middlewares platforms and business applications are being consumed as services in the cloud. [17] Cloud vendors and clients' need to maintain cloud computing security at all interfaces.

\section{INFORMATION SECURITY AND PRIVACY IN CLOUD COMPUTING}

Safety is one of the major security crisis to the users of cloud computing. Though cloud service providers demand that they provide the most reliable and secure data storage centre still they failed to give many answers to the users, such as its location, staff situation, mode of operation etc. Additionally, in a multi-tenant environment, it is very difficult for a cloud service provider to provide the level of isolation and specified dedicated environment to a single customer. Each outsourcing service providers provide service of computing or data storage for the previous service providers by an invisible way.
In order to know the shortfalls of cloud computing as well as users' security expectations in cloud computing, International Data Corporation (IDC) group (www.idc.com) conducted a survey in August 2008 and 2009 where users were asked to rate their issues and challenges experienced with cloud computing. The result illustrated that security is the biggest concern. Information security, availability and performance issues still remain in the top 3 for both years the survey was done. [6]

So, in order to protect user data each cloud computing providers are emphasizing on the use of encryption technology (such as SSL). In this way data protection can be assured but networking path, data processing and storage protection problem cannot be resolved. There are also questions about the extent to which cloud providers would accept legal responsibility and the damages that could be assessed, in the event of a proven breach of contract involving a security issue. [7]

Gartner has conducted an investigation regarding the information security issues that should be considered when dealing with cloud computing. [6].

Following lists are -

(a) Privileged access - Who has specialized/privileged access to data? So, decide a system administrator.[15] 
(b) Data location - Does the cloud vendor allow for any control over the location of data?

(c) Data segregation -By experienced professionals SLA encryption should be available at all stages.

(d) Recovery - What happens to data in case of a disaster and does the vendor offer complete restoration, and, if so, how long does the process take?

(e) Investigative support - Vendors should have the ability to investigate illegal activity.

(f) Long term validity - What happens to data if the cloud vendor goes out of business - how and in what format data will be returned?

By considering the above issues, executive provide the following suggestions to best match with cloud strategy. [6]

(a) Identification and authentication - This process is targeting at verifying and validating individual cloud users by employing usernames and passwords protections to their cloud profiles.

(b) Authorization - In authorization controls and privileges are exerted over processes flows within cloud computing. In private cloud authorization is maintained by the system administrator

(c) Confidentiality - Based on its accessibility nature specialy in public cloud confidentiality plays a major role in maintaining control over organizations' data situation at various layers of cloud applications.

(d) Availability - Availability is one of the most critical information security requirements in cloud computing. Therefore, by exploring the availability at each of the various cloud deployment and delivery models set out by the ISO, vendors and organizations can take decisions to promote a highly protected safe and sound cloud framework.

\section{METHODS TO PROVIDE DATA SECURITY IN CLOUD COMPUTING}

(a) Encrypting Files - Files must be saved in encrypted form, it can be decrypted using password only. By this process even if data is uploaded to other data centre, it can be protected.[5]

(b) Encrypting E-Mails - To enforce security programs like Hush mail can be used to automatically enrapt all the email received and sent.

(c) Use reputation service - Always go to a high reputation service provider, as they won't make the divulgence of secret data happening and newer share of the data with the marketers.

(d) Reading Privacy statement - Users must make a habit of reading precautionary statement before putting data into the cloud computing environment. Almost in every network cookies and bugs exist which can share data to other network. So, user have to make sure which data to be stored in cloud computing environment and which data is kept at personal computer.

If the data is too important that no one can be trusted to manage the clouds, enterprise can build their own private cloud. It is living inside the corporate firewall which is more secure. (e) User Filter - Some companies like Vent, Web sense and VeriTest etc. offer a system for the purpose of monitoring data. Based on content aware technology companies have to decide which data to upload or not to upload. If it finds that someone is trying to access sensitive data to the cloud, system will promptly alarm and block it.

\section{INFLUENCE OF CLOUD COMPUTING ON ELECTRONIC COMMERCE}

With the expansion of businesses, business enterprise data grow rapidly. With the fast development of networking, business enterprise data also face the threat of upcoming viruses and black guests. Cloud computing can provide data safety and business enterprises need not have to suffer from safety crisis. Cloud computing can provide a economic dependable E-commerce system, SaaS is a kind of software which generally provides this service. This system no longer requires a great deal of funds and manpower and also not requires huge establishment costs. It will be more convenient if business enterprises use network frame and application procedure in cloud environment simply by clicking on network browser.

Cloud computing reduces the establishment cost of business enterprise E-Commerce system. In order to run business function smoothly cloud purchases expensive hardware equipments with high maintenance cost. Business enterprises rent this IT infrastructure for their purpose. From this, cloud computing can provide good economic efficiency for the application of business enterprise E-Commerce.

\section{WHY CLOUD IS A GOOD PLATFORM FOR E-BUSINESS?}

(a) On-demand flexibility and scalability - The flexibility and scalability of cloud is well-suited for e-business, whether to support online marketing campaigns or other traffic spikes.[18]

(b) Increased business agility - Cloud based systems improve for example, a retailer's agility in opening new stores or locations, speed up the supply chain, and increase competitiveness, while also reducing the cost of ownership compared to a traditionally deployed, multi-channel retail system.

(c) Uptime and a smooth customer journey - At the customer interface, cloud technology can help ensure a smooth and glich-free online experience for customers. As a technology renowned for its scalability, cloud is flexible enough to cope with unexpected peaks in traffic.

In an ever more demanding and competitive retail market, cloud-based technology can help retailers give customers what they want, while getting more from them.

\section{CONCLUSION}

In today's global competitive market, companies must innovate and get the most from its resources to succeed. This requires enabling its employees, business partners, and users with the platforms and collaboration tools that promote innovation. Cloud computing infrastructures are next generation platforms that can provide tremendous value to companies of any size. They can help companies achieve more efficient use of their IT hardware and software 
investments and provide a means to accelerate the adoption of innovations. Cloud computing increases profitability by improving resource utilization. Costs are driven down by delivering appropriate resources only for the time those resources are needed. Cloud computing has enabled teams and organizations to streamline lengthy procurement processes.

Cloud computing is still a very young technology and we still having more room for improvement. Although the meaning of cloud computing may be differ from one point of view of a person to another, it still all boils down to sharing one meaning which is - delivering information over the internet. [19]

\section{REFERENCES}

[1] Wang D, (May,2013), "Influences of Cloud Computing on E-Commerce Businesses and Industry", Journal of Software Engineering and Applications, Vol. 6, pp. 313-318

[2] Leavitt N, (2009), "Is Cloud Computing Really Ready for Prime Time?", Computer, Vol. 42, pp. 15-20.

[3] Weinhardt C, Anandasivam A, Blau B, and Stosser J, (2009), "Business Models in the Service World", IT Professional, Vol. 11, pp. 28-33

[4] Gens F, (2009), "New IDC IT Cloud Services Survey: Top Benefits and Challenges", IDC eXchange, viewed 18 February 2010

[5] Juncai S and Shao Q, (June, 2011), "Based on Cloud Computing E-commerce Models and Its Security", International Journal of e-Education, e-Business, eManagement and e-Learning,Vol. 1, No. 2

[6] Ramgovind S, Eloff MM, Smith E, (2010), "The Management of Security in Cloud Computing", IEEE

[7]Li H, Sedayao J, Jimison E, Spence C and Chalal S, (January, 2009), "Developing an Enterprise Cloud Computing Strategy", White paper, Intel Information Technology, Computer Manufacturing, Cloud Computing

[8] J. Li and J. Z. Liu, (December, 2010), "Influence of Cloud Computing on Educational Informationization of China Rural Areas", The Proceedings of Information Science and Engineering Conference, Hangzhou, 4-6 December, 2010,pp. 281-283.
[9] F. Kashefi, M. Majd, M. Darbandi, H. Purhosein, K. Alizadeh and O. Atae, (2011), "Perusal about Influences of Cloud Computing on the Processes of These Days and Presenting New Ideas about Its Security", The Proceedings of the 5th International Conference on Application of Information and Communication Technologies (AICT), Baku, 12-14 October 2011, pp. 1-4.

[10] S. Qin, (2011), "What Will Cloud Computing Provide for Chinese M-Learning?", The Proceedings of International Conference on E-Education, Entertainment and E-Management, Bali, 27-29 December 2011, pp. 171-174.

[11] S. L. Lai, (January), "The Influences of Cloud Computing to the Traditional Software Project and Our Corresponding Strategies", The Proceedings of the $3 \mathrm{rd}$ International Conference on Intelligent System Design and Engineering Applications, Hong Kong, 16-18 January 2013, pp. 1461-1464.

[12] H. X. Zhang, (July), "Research on the Influence of Cloud Computing on the Virtual Operation Performance Management", The proceedings of the 7th International Conference on Computer Science \& Education, Melbourne, 14-17 July 2012, pp. 235-238.

[13] Dooley B, (2010), “Architectural Requirements Of The Hybrid Cloud", Information Management Online, viewed 10 February 2010

[14] Global Netoptex Incorporated , (2009), "Demystifying the cloud. Important opportunities, crucial choices"

[15] Brodkin J, (2008), “Gartner: Seven cloud-computing security risks",Infoworld, viewed 13 March 2009

[16] ISO. ISO 7498-2:1989. Information processing systems- Open Systems Interconnection. ISO 7498-2

[17] Klems, M, Lenk, A, Nimis, J, Sandholm T and Tai S, (2009), 'What's Inside the Cloud? An Architectural Map of the Cloud Landscape', IEEE Xplore, pp 23-31, viewed 21 June 2009.

[18]"E-business and the cloud Is cloud computing the right fit for your online business?", White Paper E-business and the cloud, www.claranet.co.uk

[19] D. Jin and S. Lin, (2012), "Research of E-Commerce Based on Cloud Computing", CSIE, Vol. 2, pp. 15-20 